\title{
Contributed Papers for JD19: Abstracts
}

\section{Complex of Meteoroid Orbits with High Eccentricities}

Svitlana V. Kolomiyets \& Boris L. Kashcheyev (Kharkiv National University of Radioelectronics, Ukraine)

\begin{abstract}
In our work is demonstrated the method that can help to predict the existence of distant objects in the Solar system. This method is connected with statistical properties of a heliocentric orbital complex of meteoroids with high eccentricities. Heliocentric meteoroid orbits with high eccentricities are escape routes for dust material from distant parental objects with near-circular orbits to Earth-crossing orbits. Ground-based meteor observations yield trajectory information from which we can derive their place of possible origin: comets, asteroids, and other objects (e.g. Kuiper Objects) in the Solar system or even interstellar space [Kolomiyets, S. 2002, Abstracts ACM 2002, Berlin, 371; Kashcheyev, B. Kolomiyets, S. 2001, SP-495, 643]. Such information is important at planning space missions. We analyze elliptic meteor orbits with high eccentricities that were registered in 1972 - 1978 in Kharkiv (Ukraine). Statistical distributions of radius-vectors of nodes, and aphelia of orbits of meteoroids contain key information about position of greater bodies. We discuss the accuracy of the ground-based radar observations of faint meteors. An estimation of selectivity factors of meteor radar measurements is made on base of radiophysical and astronomical researches of meteoric substance and its interaction atmosphere of the Earth.
\end{abstract}

\section{Meteor Showers in 1993 and 1998: On the Goniometric Data}

Sergei A. Kalabanov, Vladimir V. Sidorov, Tamara K. Filimonova \& Jury N. Kosenkov (Kazan State University, Russia)

\footnotetext{
Abstract. On the basis of application of a new discrete quasy-tomography approach of analysis to the goniometric data of the Kazan meteor radar it were examined acts of known meteor showers working during six months of continuous observation in 1993 and 1998 years.

The angular resolution of coordinates of radiants for all measured data is $2^{\circ} \times 2^{\circ}$. For intensive meteor showers the resolution achieved $1^{\circ} \times 1^{\circ}$. An average heliocentric velocity of a shower was calculated by averaging individual velocities after operation of an identification of a concrete set of measured data with an investigated meteor shower. Most stable annual meteor shower GEMINIDS was used as a master shower.

It is revealed, that many weak meteor showers having personal names and which should be observed within half-year in 1993 and 1998, have not detected with expected parameters. Others however were observed as though casual showers which operated within several days in immediate vicinity to expected ones, but not repeating in the same dates and with identical parameters.
} 
The measured parameters of orbits of observed meteor showers are resulted in comparison with known lists of the basic meteor showers for northern celestial hemisphere.

\title{
Sporadic Meteor Microshowers: Data And Parameters
}

Vladimir V. Sidorov, Sergei A. Kalabanov \& Rashid A. Ishmuratov (Kazan State University, Ukraine)

\begin{abstract}
A development of a new quasy-tomography technology of processing of the goniometric data from a meteor radar, allowed considerably to increase the angular resolution of radar measuring and its application to the data received at continuous observation within six same months 1993 and 1998 on meteor radar at KSU, has allowed to find out new astronomical objects sporadic microshowers and to measure parameters of their orbits.

We have named microshowers as sets of the registered meteors having close velocities and their coordinates of radiants, acting during one or more days, but not repeated in identical hours and dates in two different years.

Possible sources of occurrence of such formations are discussed:
\end{abstract}

- Dust traces of small comets and the asteroids which have not been found out yet by optical astronomical tools.

- Dust traces of large meteoroids, fallen to the Earth as bolides or flown by in immediate vicinity to the Earth at the moment of time, close to the moments of display of microshowers.

- The groups of the meteor particles which have been pulled out from bodies of meteor streams by consecutive gravitational perturbation of planets, with their preserved collective properties during evolution.

\section{Meteor Researches at KHNURE}

Svitlana V. Kolomiyets, Yuri I. Voloshchuk, Boris L. Kashcheyev \& Nikolay I. Slipchenko (Kharkiv National University of Radioelectronics, Ukraine)

Abstract. The Scientific-Educational Center of Radioengineering of the Kharkiv National University of Radioelectronics (KHNURE) is one of the oldest radar meteor centers, which was founded by B. L. Kashcheyev in 1958. The first automatic meteor radar system in Ukraine "MARS" is connected with our University. There are long-term observational series of meteor rates and orbital data in the Center. Fields of the KHNURE researches are: a structure of meteor showers, a determination of meteoroid orbits, an influx of cosmic rubbish in the Earth atmosphere, search of parental bodies of meteoroids, a statistic analysis of measurement results of radiometeors, an estimation of errors of meteor radar measurements, a search for real hyperbolic orbits and interstellar meteoroids. KHNURE disposes a unique electronic orbital catalogue. This catalogue contains the primary information, velocities, radiants and orbits of nearly 250,000 radiometeoroids with masses from 0.001 to $0.000001 \mathrm{~g}$. The "MARS" registered these data during observations of 1972 - 1978. From these data 5160 meteor streams are singled out. New classification of streams is made in view of their 
structure. The study of meteor stream orbits from the KHNURE data bank allow to predict orbits of a big number of undiscovered "dangerous" NEOs.

\title{
Fluctuations in the Activity Curve of the 2002 Leonids
}

Lola Herrera Ruiz, Luis R. Rubio Bellot \& Miquel Serra-Ricart (Instituto de Astrofisica de Canarias, Tenerife, Canarias, Spain)

\begin{abstract}
We present observations of the 2002 Leonid meteor shower taken with four intensified video cameras from Teide Observatory (Tenerife, Spain) on November 19, 2002. The cameras, fitted with f/1.4, $85 \mathrm{~mm}$ objectives, were aimed at $6^{\circ}$ above the horizon in order to monitor the largest atmospheric volume possible. The most sensitive camera detected 1300 meteors between 0312 and 0456 UT, i.e., the period covering the European Leonid storm of 2002 . The activity curve constructed from these data peaks at 0401 UT, about 9 minutes earlier than indicated by the visual observations analyzed by the International Meteor Organization. Our results thus favor the model of Lyytinen and van Flandern, who predicted the first maximum at 0402 UT. We find statistically significant oscillations in the activity curve. A Fourier analysis delivers a period of about 7 minutes. These observations confirm the discovery of Singer et al. (2000, AGU Spring Meeting Abstracts, 41, 21) that density fluctuations exist in the Leonid dust trails at spatial scales of $10000-30000 \mathrm{~km}$. The oscillations we observe are very similar to those detected by Singer et al., the only difference being that the dust trail is 132 years older.
\end{abstract}

\section{Geophysical Aspects of Dergaon Meteorite}

Kalpana Duorah, Sarat Phukan, Parag Phukon, Amulya Mazumdar \& Hira L. Duorah (Physics Department, Gauhati University, India)

\begin{abstract}
The Astrophysics group, Gauhati University under the supervision of Dr. Kalpana Duorah collected a meteorite sample in Dergaon, Assam India having geographical location 9352/ E longitude and 26041/50 "N latitude. The sample is registered by the International Meteoritic Society in their Bulletin (May 2001) as Dergaon meteorite. Preliminary analysis showed that the parent meteorite had total mass of about $50 \mathrm{~kg}$ out of which the collected sample was determined to be $10.2 \mathrm{~kg}$. This is found to be a stony meteorite of group H 5. Electron probe Microanalysis of the sample revealed that the dominant minerals are olivine and pyroxene and subordinate amount of metal phases. The whole rock analysis has produced the element ratios $\mathrm{Fe} / \mathrm{Si}=0.80$ and $\mathrm{Si} / \mathrm{Mg}$ $=1.074$. and the trace elements and the rare earth elements are obtained by the inductively coupled plasma mass spectrometry. The paper provides detailed analysis of the Dergaon Meteorite.
\end{abstract}

\section{Meteorite Physical Properties: Implications for Asteroids}

George J. Flynn (State University of New York, Plattsburgh, NY, USA)

\begin{abstract}
Meteorites are believed to be samples of asteroids. We measured the porosity of carbonaceous and ordinary chondrite meteorites by He pycnometer and imaged the porosity by computed microtomography. We found fresh samples of most ordinary chondrites have $\sim 5 \%$ to $15 \%$ porosity, while carbonaceous chondrites have even higher porosity (up to $30 \%$ in Allende). This porosity occurs in three forms: extended cracks, vugs, or gaps between the matrix and
\end{abstract}


the chondrules. We performed hypervelocity impact studies on chondritic meteorites to determine how the presence of chondrules in a more porous matrix effects fragmentation. The gaps between the chondrules and the matrix appear to enhance the ejection of whole chondrules or large chondrule fragments during hypervelocity impact, while much of the debris significantly smaller than the chondrules is chemically-distinct matrix material. We measured the speed of sound, bulk modulus, and elastic modulus of several ordinary chondrite meteorites. The speed of sound ranges from $1,100 \mathrm{~m} / \mathrm{sec}$, in a fragment of Saratov, an extremely friable ordinary chondrite, up to $4,000 \mathrm{~m} / \mathrm{sec}$ in more compact ordinary chondrites. The weakest meteorites exhibit physical properties more comparable to volcanic tufts than to compact basalts. Modeling of stone asteroids needs to consider a wide range of physical Properties.

\title{
Cometary Features of Newly Discovered Bodies
}

Milos Tichy, Jana Ticha \& Michal Kocer (Klet Observatory, Czech Republic)

\begin{abstract}
The majority of new ground-based discoveries of comets comes from large surveys. The first step of exploration of newly discovered cometary bodies consists of the confirmatory astrometric observation and also detection of their cometary features. Only some of both professional and amateur stations do a preliminary analysis of cometary activity of a particular newly discovered body. A timely recognition of cometary features of a particular body having an unusual orbit can help in planning further observing campaigns. One of main goals of the Klet Observatory NEO astrometric follow-up program is an analysis of possible cometary activity of newly discovered unusual bodies. We present here the first results obtained with our new 1.06-m KLENOT Telescope, which we put into operation in March 2002. The CCD camera used on this telescope enables detection of comet's gas, plasma and dust activity in a more satisfactory way.

Acknowledgments: This work was supported by grants MSMT LA163 and GACR $205 / 02 / \mathrm{P} 114$.
\end{abstract}

Observations and Investigation of Comets in Kyiv University Klim Churyumov (Kyiv Shevchenko National University, Kyiv, Ukraine)

\begin{abstract}
The following results of observations and investigation of comets in Kyiv Natsional Shevchenko University are discussed: a) Narrow band photometry comets, peculiarities of comet nuclei gas production rates, taxonomy of comets; b) Optical spectroscopy of comets, identification of emission lines in comet spectra, physical parameters of the comet neutral gas atmospheres, detection of the luminescent continuum in comet spectra: c) Near nucleus phenomena in comets, the dust shells in comet C/1995 O1 (Hale-Bopp); d) Large scale phenomena in comets and their theoretical modelling; e) Light curves of comets and their connection with he solar activity.
\end{abstract}

\section{Investigation of the Comet 19P/Borrelly Spectra}

Klim I. Churyumov, Igor V. Lukýanyk \& Larisa S. Chubko (Kyiv National Shevchenko University, Ukraine)

Abstract. Several hundreds of spectra and monochromatic images of Comet 19P/Borrelly were obtained with the Multi Pupil Fiber Spectrograph (MPFS) 
installed in the Prime Focus of the 6-m telescope of the SAO of the RAS (Nizhny Arkhyz) on Aug. 13 and 15, 2001, and with Spectral Camera with Optical Reducer for Photometrical and Interferometrical Observations (SCORPIO) installed in the Prime Focus of the 6-m telescope of the SAO of the RAS on August 16 and 17. The observations were made 41-37 days before the New Millennium DS-1 probe approach with the comet nucleus. For detailed identification we selected the spectrum of the comet 19P/2000 Borrelly, obtained Aug. 15, 2001. The rates of gas production $(\log Q)$ of neutral molecules from the nucleus of the comet were calculated using Haser's model for Aug.17, 2001: $\log Q(\mathrm{CN})$ $=25.48, \log Q\left(\mathrm{C}_{3}\right)=24.29$ and $\log Q\left(\mathrm{C}_{2}\right)=24.97$.

\title{
Physical Parameters of the Comet Ikeya-Zhang Atmosphere
}

Klim I. Churyumov, Igor V. Lukýanyk, Alexey A. Berezhnoy, Vahram H. Chavushyan, Larisa S. Chubko, Valery V. Kleshchonok, Lourdes Sandoval \& Alejandro Palma (Kyiv National Shevchenko University, Ukraine)

\begin{abstract}
Several middle-resolution optical spectra of Comet C/2002 C1 (Ikeya-Zhang) were obtained on May 5, 2002 with the help of the $2.12-\mathrm{m}$ reflector of the Guillermo Haro Astrophysical Observatory. On the basis of the intensity distribution along the slit of the spectrograph in $\mathrm{C}_{2}, \mathrm{C}_{3}, \mathrm{CN}$ emission lines we determined the velocities of expansion $(v)$ and life times $(\tau)$ of these molecules: for $\mathrm{C}_{2}(5165 \AA)\left[201 \mathrm{~m} / \mathrm{s}, 3.08 \times 10^{6} \mathrm{~s}\right]$; for $\mathrm{C}_{3}(4050 \AA)[166 \mathrm{~m} / \mathrm{s}$, $\left.0.075 \times 10^{6} \mathrm{~s}\right]$ and for $\mathrm{CN}(4200 \AA)\left[157 \mathrm{~m} / \mathrm{s}, 0.06 \times 10^{6} \mathrm{~s}\right]$.
\end{abstract}

\section{Changes in the Spectra of Comet C/1999 S4 in July, 2000}

Larisa S. Chubko (Vinnytsia Pedagogical Kotsyubynsky University, Ukraine), Klim I. Churyumov, Igor V. Lukýanyk \& Ayyub S. Guliev (Kyiv National Shevchenko University, Ukraine)

\begin{abstract}
The spectra of comet C/1999 S4 (LINEAR) were obtained with the eshelle-spectrograph (and CCD) installed on the 2-m Zeiss reflector of the Shamakhy Astronomical Observatory of the Azerbajan Academy of Sciences (Mount Pirkuli) on July 21 and 23, 2000. The spectra of comet C/1999 S4 (LINEAR) were also obtained with the UAGS spectrograph (long slit and CCD) installed on the 1-m Zeiss reflector of the SAO of the RAS (Northern Caucasus, Nizhny Arkhyz) on July 23, 27 and 28, 2000. Emissions lines of the molecules $\mathrm{C}_{2}, \mathrm{C}_{3}, \mathrm{CN}, \mathrm{NH}, \mathrm{CH}, \mathrm{NH}_{2}, \mathrm{CO}, \mathrm{CO}^{+}, \mathrm{H}_{2} \mathrm{O}^{+}$and other were identified in the spectra of comet C/1999 S4 obtained on July 21, 23 and 27, 2000 during splitting of the cometary nucleus. Changes in the spectra are discussed.
\end{abstract}

\section{Peculiarities of Light Curves of Some New Bright Comets}

Vitaly S. Filomenko \& Klim I. Churyumov (Astronomical Institute of Kharkov National University, Ukraine)

\footnotetext{
Abstract. The light curves of ten comets [C/2000 SV74 (LINEAR), C/2000 WM1 (LINEAR), C/2001 A2 (LINEAR), C/2001 OG108 (LONEOS), $\mathrm{C} / 2002 \mathrm{C} 1$ (Ikeya-Zhang), C/2002 F1 (Utsunomiya), C/2002 O4 (Hoenig), $\mathrm{C} / 2002 \mathrm{~V} 1$ (NEAT), C/2002 X5 (Kudo-Fujiakawa) and 19P/ Borrelly] were constructed on the basis of visual physical observations of these comets. Photometric parameters $H_{10}$ (Vsekhsvyatskii's absolute magnitude), $H_{y}$ (absolute
} 
magnitude) and $n$ (photometric parameter that characterizes the rate of change of cometary brightness with heliocentric distance) were determined. The analysis of light curves showed that the parameter $n$ of seven comets [C/2001 A2, $\mathrm{C} / 2001$ OG108, C/2002 C1, C/2002 F1, C/2002 O4, C/2002 V1 and 19P] undergoes abrupt changes. We found that these jumps in the rate of change of the cometary brightness were correlated with outbursts of comets. The statistically significant (reliability $>0.95$ ) influence of the phase dependence on the visual light curves for three comets had been found. The values of phase coefficient $(\beta)$ for these comets were determined: (1) $\mathrm{C} / 2001 \mathrm{~A} 2: \beta=0.086 \pm 0.027$ $\mathrm{m} /$ degree (before perihelion), (2) $\mathrm{C} / 2001$ OG108: $\beta=0.032 \pm 0.025 \mathrm{~m} /$ degree (before perihelion) and $0.052 \pm 0.016 \mathrm{~m} /$ degree (after perihelion), (3) $\mathrm{C} / 2002 \mathrm{~V} 1$ : $\beta=-0.062 \pm 0.010 \mathrm{~m} /$ degree (before perihelion).

\title{
Spatial Distribution of the Halley Comet Dust Continuum from Data of TKS - VEGA 2
}

Penka (Stoeva) Muglova, V. Guineva \& R. Werner (Central Solar - Terrestrial Influences Laboratory, Bulgarian Academy of Sciences, Bulgaria)

\begin{abstract}
Spatial distribution of the solar light scattered by dust particles in Halley comet coma towards the Sun and in the near tail is investigated using spectra measured by TKS, VEGA 2. Monochromatic composed images in 5260 Åare constructed from consequently registered two-dimensional images for 9 and 10 March 1986. Radial profiles presenting the intensity as a function of the projected distance to the nucleus $p$ are also used in the research.

Two strong jets are observed. One of them is in direction towards the Sun and expands up to $p=19500 \mathrm{~km}$ from the nucleus, and the other is perpendicular to it and reaches $p=19000 \mathrm{~km}$. The weaker slope of the radial profile near the nucleus is explained by the greater optical depth of the inner coma or by the quick change of the scattering angle.

In antisolar direction, in the near tail, a weaker intensity of the dust continuum is observed. The intensity increasing, obtained far from the nucleus, and the radial profile maxima are probably connected with a dust shells formation due to jet activity and nucleus rotation.
\end{abstract}

\section{Phase Dependencies of Cometary Visual Light Curves}

Vitaly S. Filomenko \& Klim I. Churyumov (Astronomical Institute of Kharkov National University, Ukraine)

\footnotetext{
Abstract. Generally, the integrated flux produced by a comet on Earth is the sum of the fluxes produced by gas and dust in the cometary atmosphere and by the solid cometary nucleus. The contribution from the nucleus to the integrated comet brightness is extremely small and it can be ignored. The contributions from the gas and dust are functions of the comet heliocentric distance. But the flux from dust also depends on the phase angle. Therefore, the phase dependence of brightness can be determined in principle from the visual integrated light curves of comets whose dust-scattered radiation accounts for a considerable fraction of the total radiation. The statistical significant influence of phase dependencies on the light curves of eight comets had been found. The values of phase coefficient of these comets had been determined. For three comets the values of phase coefficient are negative. For other five comets the mean value of phase coefficients is $0.047 \mathrm{~m} /$ grad that practically coincides with mean value of phase coefficients of C-type asteroids $(0.041 \mathrm{~m} / \mathrm{grad})$. Apparently the
} 
phase dependencies of these comets determines by dark carbon particles in their atmospheres.

\title{
Infrared Observations in the 3-Micron Region of Comets
}

Karen Magee-Sauer, Michael J. Mumma, Neil Dello Russo, Michael A. DiSanti \& Erika L. Gibb (Rowan University, NJ, USA)

\begin{abstract}
The 3-micron spectral region is rich in emissions from key cometary volatile species. Spectral lines of several molecules are identified in this region, including $\mathrm{C}_{2} \mathrm{H}_{2}, \mathrm{HCN}, \mathrm{NH}_{3}, \mathrm{H}_{2} \mathrm{O}$ ("hot-band" emission), $\mathrm{OH}$ (prompt emission and fluorescent emission), and $\mathrm{NH}_{2}$. These species are key to understanding several important questions in cometary science. Acetylene is an important link to the origin and processing of the natal ices in comets. The abundance of HCN and its release from the nucleus provides important information on the processing of nuclear ices from radiation and gas-phase chemistry, as well as delivery of biogenic molecules to Earth. As the fully reduced form of nitrogen, $\mathrm{NH}_{3}$ is the key species for understanding the nitrogen chemistry of comets and thus chemistry in the region of comet formation. OH ("prompt") and $\mathrm{H}_{2} \mathrm{O}$ emission in the spectrum provide a means of measuring water production directly. Here, we present spectra, rotational temperatures, spatial profiles, production rates, and relative abundances of molecules detected in the 3-micron region in observed comets from Hyakutake to the present. Results obtained through the CSHELL instrument at the NASA IRTF and the significant gains obtained through use of the NIRSPEC instrument at the Keck Observatory will be presented.
\end{abstract}

\section{Results From the UMD Physical Properties of Comets Survey}

Carey M. Lisse, Michael F. A'Hearn \& Yanga R. Fernandez (University of Maryland, MD, USA)

\begin{abstract}
We report on an ongoing statistical study of the emitted dust and exposed nuclei of a survey of the brightest near-Earth comets over the last 13 years. Combined thermal infrared and optical observations are analyzed using dynamical, spectral, and morphological coma models (Lisse et al. 1998, ApJ, 496, 971; Lisse et al. 1999, Icarus, 140, 189; Fernandez et al. 2000, Icarus, $147,145)$ to update and improve dust emission rates (Kresak \& Kresakova 1987, in Symposium on Diversity and Similarity of Comets, ESA SP-278, 739) and nucleus size estimates (Jewitt 1991, in Comets in the Post-Halley Era, eds. R. L. Newburn, M. Neugebauer, \& J. Rahe, Kluwer Academic, Dordecht, 19).

Using these results, we show that 1) there is more than enough dust emitted from short period comets into bound solar system orbits to create and support the current interplanetary dust cloud (IPD);2) that a population of dormant or extinct comets in the solar system is quite plausible; and 3) that the lifetime versus sublimation for the short period comets is much longer than their dynamical lifetime.
\end{abstract}

Deep Impact Mission Target Comet Nucleus Characterization

Karen J. Meech, Jana Pittichova, Henry Hsieh, Yan Fernandez, James M. Bauer, 
Michael J. Belton, Michael F. A'Hearn, Olivier R. Hainaut, Hermann Boehnhardt \& Gian-Paolo Tozzi (Institute for Astronomy, Hawaii, USA)

\begin{abstract}
Comet 9P/Tempel 1 is the target of the 8th NASA Discovery Mission, Deep Impact. The focus of the ground-based observing program has been to characterize the nucleus for mission planning. We have obtained data over a full apparition from 11/97 through the present on a total of over 200 nights using 16 telescopes worldwide. The nearly 2000 images represent over 250 hours of integration. We will present the, nucleus size, heliocentric light curve, and Afrho values vs. time, and report on the status of the analysis of the rotation period. We will report on the color of the nucleus and dust coma (using BVRI filters) as a function of heliocentric distance. We have analyzed the data as a function of phase angle to infer the nucleus surface scattering properties. The comet is quite phase darkened, with a linear phase function of $0.07 \mathrm{mag} / \mathrm{deg}$, and a negative value of G-parameter suggestive of a low albedo rough surface. We will also present a series of enhanced images to search for evidence of features (e.g. jets and other structures) in the dust coma.

Acknowledgements: Support provided by NASA Grants NAGW-4495, NAGW5015, NAG-54080, and NAG-59006 and through Univ. of MD subcontract Z667702 (NASA contract NASW-00004).
\end{abstract}

\title{
Korean NEO Station in South Africa
}

Wonyong Han, Yong-Ik Byun, Hong-Kyu Moon, Yong-Woo Kang, Hong-Suh Yim, Sunyoup Park, Young-Ho Bae \& Sung Yeol Yu (Korea Astronomy Observatory, Korea)

\begin{abstract}
The joint Near Earth Object (NEO) project team of Korea Astronomy Observatory (KAO) and Yonsei University Observatory (YUO) has recently installed a 0.5 -meter robotic telescope at the Sutherland Observatory in South Africa. This telescope with 2-degree FOV is operated in fully automated mode, making daily reports on discoveries NEOs and other significant luminosity variability via internet. The KAO-YUO joint team plans to install similar facility in Australia and Chile soon, making a network of survey telescopes for southern hemisphere. In spite of its small aperture size, this network will be an important tool in identifying southern NEOs, especially those in the form of comets. This paper summarizes the observatory system, data handling, and our parallel efforts to characterize NEOs with follow-up light curve investigation.

Acknowledgments: The joint project is funded by the National Research Laboratory Program of Korean Ministry of Science and Technology (KAO) and by the Korean Research Foundation.
\end{abstract}

\section{Observations of 2002 NY40: An Ordinary Chondrite?}

Ellen S. Howell, Andrew S. Rivkin, Michael C. Nolan, Jean-Luc Margot, Gregory Black, Schelte J. Bus, Michael Hicks, William T. Reach, H. T. Jarrett \& Richard P. Binzel (Arecibo Observatory, Cornell University, USA)

Abstract. The near-Earth asteroid 2002 NY40 was discovered on July 14 ,
2002 by the LINEAR survey. The object made a close pass by the Earth on
August 18, when observations were obtained at a large range of wavelengths,
from visible to radar $(12.6 \mathrm{~cm})$. The combination of visible and near-infrared 
spectroscopy gives some indication of the composition. Thermal emission in the 3 -micron region gives constraints on the visible albedo, which are confirmed independently by the radar size and visible magnitude. 'The lightcurve was well measured by a large number of observers, and the rotation period is welldetermined at 19.99 hours. The spectrum and albedo are a very good match to an LL chondrite spectrum over the range measured. No appreciable reddening is seen in the asteroid spectrum, which suggests that the surface has not been noticeably affected by the same processes seen in many other pyroxene and olivine-rich asteroids. The radar images show that this asteroid looks like two spheroidal units joined together. Analysis and implications of these observations will be discussed.

\title{
Database for Geophysical and Geological Properties of NEOs
}

W. F. Huebner (Southwest Research Institute, San Antonio, TX 78228-0510, USA)

\begin{abstract}
Collisions of asteroids and comets with Earth have occurred in the past. About 150 impact structures on the Earth's continents have been identified. There may be many more impacts whose structures have eroded over time. Asteroids and comets still collide with the planets and will again collide with the Earth. These are random events. No asteroid is now known to be on a collision course with the Earth and we do not know when the next catastrophic impact might occur. Many parameters are needed to develop credible mitigation or collision avoidance strategies. We know little about geophysical properties of NEOs. Here we report on progress on the database for geophysical and geological properties of NEOs and their analog materials. The database consists of four parts: An observational database, a material properties database, a database for mission and instrument development, and a database useful for dissemination of projects and results and for public outreach. The database will be available at: http://neodata.space.swri.edu/. For atomic and molecular data see: http://amop.space.swri.edu/.
\end{abstract}

\section{The Dynamic Estimation of the Mass of the Main Asteroid Belt}

Elena Pitjeva (Institute of Applied Astronomy of Russian Academy of Science, Russia)

\begin{abstract}
Perturbations from asteroids affect significantly on the orbits of the inner planets and should be taken into account when high-accuracy planetary ephemerides are constructed. On the other hand, from an analysis of the motions of the major planets by processing of precise measurements of ranging to the Viking, Pathfinder, Mars Global Surveyor spacecraft and planets (1961-2002) some physical parameters of the asteroids have been obtained. The masses of several the biggest asteroids (Ceres, Pallas, Vesta, Juno) have been determined individually, masses of the most relevant 296 asteroids have been derived from their latest published diameters based on IRAS data and observations of occultations of stars by minor planets, making use of the corresponding densities. The total contribution of all remaining small asteroids is modeled as an acceleration caused by a solid ring in the ecliptic plane. As a sequence the total mass of the main asteroid belt was obtained: $M=(14 \pm 2) \times 10^{-10}$ mass of Sun. $n$ derived expression for estimating the total number of minor planets in any unit interval of absolute magnitude $\mathrm{H}$ was compared with the observed distributions of the
\end{abstract}


asteroids (52224 numbered, 152451 unnumbered) and distribution of the SAM model by Tedesco et al.

\title{
ISO Observations: Results on Asteroids
}

Elisabetta Dotto, Maria A. Barucci, John R. Brucato, Thomas G. Mueller \& Marcello Fulchignoni (INAF-Osservatorio Astronomico di Roma, Italy)

\begin{abstract}
We carried out observations of 16 asteroids with different ISO instruments (PHT-P, PHT-S and SWS) obtaining low resolution spectra up to 12 micron, high resolution spectra up to 45 micron and spectrophotometric data up to 60 micron. The aim of these observations was to investigate the physical properties of the surface of these bodies, and to improve our understanding on the processes which governed their formation and evolution. We interpreted the obtained data in terms of asteroid surface composition, comparing the observed spectra with laboratory reflectance spectra of selected mineral, mineral mixture and meteorite particulates of different grain sizes. The characterization of the material on the asteroid surfaces (presence, abundance and/or composition of minerals or chemical species) was based on the interpretation of observable diagnostic spectroscopic properties. The obtained results will be presented and discussed.
\end{abstract}

\section{ESO-VLT Large Program on Centaurs and TNOS}

Maria Antonietta Barucci, Hemann Boehnhardt \& Project Team (Observatoire de Paris, LESIA, France)

\begin{abstract}
The ESO Large Program on the study of Trans-Neptunian Objects (TNOs) and Centaurs has been carried out during the last two years (April 2001-March 2003) at the Paranal (Very Large Telescope VLT) and La Silla (New Technology Telescope NTT) observatories of the European Southern Observatory (ESO) in Chile.

The aim of the Large Program was to investigate the physical properties of this population composed of cold and primitive objects.

Results has been obtained for multi-wavelength imaging, BVRI and JHK filters of 70 and 30 objects respectively, as well as for low-dispersion visible and near-IR spectroscopy of about 12 objects. The photometric results and analyses of the relative correlations between colours, photometric gradients and dynamical properties of the objects will be presented and discussed. The spectroscopic results have been interpreted with models computed using geographical mixture of organics, minerals and ices in order to constrain the surface composition. The spectra show a wide range of slopes, some are featureless with almost constant gradients over the visible-NIR range, while others show absorption signatures of ices.

The limit and the utility of the ground-based observations will be discussed together with the necessity of a space exploration.
\end{abstract}

Deep TNO Search Near Invariable Plane using Subaru Telescope Daisuke Kinoshita, Jun-ichi Watanabe, Naotaka Yamamoto, Tetsuharu Fuse, 
Seidai Miyasaka, Kyoko Muroi \& An-Li Tsai (National Astronomical Observatory of Japan, Japan)

Abstract. We have carried out deep imaging observation near the invariable plane using Subaru telescope with its prime focus camera "Suprime-Cam". We have integrated single field of $0.2 \mathrm{sq}$. deg. deeply on 5,6 October 2002, and followed up detected objects on 3 December 2002. SDSS r'-band filter was used for this observation to maximize the number of detection. The limiting magnitude is $r^{\prime}=28.0$ for signal-to-noise ratio of 5 . Assuming typical color of TNO, it roughly corresponds to $R=27.7 \mathrm{mag}$. The aim of this observation is (1) to investigate existence or non-existence of distant TNO beyond $50 \mathrm{AU}$ from the Sun and (2) to explore the faint-end of the luminosity function. All the detected TNOs have heliocentric distances ranging $38<R_{H}<45 \mathrm{AU}$. We will report preliminary results of our deep survey.

\title{
Physical Characteristics of Centaurs and TNOs
}

Alain Doressoundiram, Maria A. Barucci \& Nuno Peixinho (Observatoire de Paris, France)

\begin{abstract}
Beyond the orbit of Neptune exists a population of bodies remaining from the formation of the solar system. These are the Kuiper Belt or Trans-Neptunian Objects (TNOs). Scientific interest in these bodies arises because this region of the solar system may preserve some of the most primitive materials available to direct investigation.

We have started since 1997 a multicolor photometric survey with the aim of collecting a large and homogeneous set of color data for TNOs and Centaurs objects. With this large dataset obtained mostly at CFHT and ESO, we performed relevant statistical analyses to search for compositional structures, interrelations with related populations and correlations with physical and orbital parameters.

One of the most puzzling features of the Kuiper Belt is the optical color diversity that seems to prevail among the observed TNOs. In this work, we will present results on the strong correlations found with some orbital parameters $(i$, $e, q)$ for the Classical Kuiper Belt. The correlations found are important because they are diagnostic of some physical effects processing the surfaces of these icy and primitive objects. We will give a global picture of the color properties and trends within the Kuiper Belt.
\end{abstract}

\section{The Albedo f Trans-Neptunian Object 1579 (1993SC)}

Iwan P. Williams \& Alan Fitzsimmons (Queen Mary, University of London, UK)

Abstract. ISO observations of the infrared emission from the Trans-Neptunian object 15789 (1993SC) were obtained in 1997 and have been published (Thomas et al. 2000, ApJ, 534, 446). Determination of the albedo, and hence the radius also requires a knowledge of the reflected (visible) radiation. In their publication, Thomas et al used ground based data obtained in 1995. Since that time ground based observations close in time to the ISO observations have been ob- 
tained. The paper presents the new observations and deduced albedo based on these observations.

\title{
Detectability of Lightcurves of KBOs
}

Pedro Lacerda \& Jane Luu (Leiden Observatory, Leiden, The Netherlands, \& Harvard-Smithsonian CfA, Cambridge, MA, USA)

\begin{abstract}
We present a statistical study of the detectability of lightcurves of Kuiper Belt objects (KBOs). Some Kuiper Belt objects display lightcurves that appear "flat", i.e., there are no significant brightness variations within the photometric uncertainties. The lack of brightness variations may be due to (1) the objects have very nearly spherical shapes, or (2) their rotation axes coincide with the line of sight. We investigate the relative importance of these two effects and relate it to the observed fraction of "flat" lightcurves. This study suggests that the fraction of KBOs with detectable brightness variations may provide clues about the shape distribution of these objects. Although the current database of rotational properties of KBOs is still insufficient to draw any statistically meaningful conclusions, we expect that, with a larger dataset, this method will provide a useful test for candidate KBO shape distributions.
\end{abstract}

\section{Frozen Volatiles in the Icy Satellites and KBO Objects}

Jacek Leliwa-Kopystynski (Warsaw University, Physics Dept., Institute of Geophysics, Poland)

\begin{abstract}
The first physical assumption of this work is that the volatiles were transported to the growing icy/mineral objects of the Outer Solar System by means of accretion. The relative amounts of different volatiles in the accreting matter depends on the local temperature of that matter therefore, roughly, on the solar distance. The second assumption concerns degassing of the volatiles: gases instantaneously escape from the growing body if temperature of accreting matter in the moment of an impact is enough high. Discussion of the conditions related to presence of pristine-originated solidified volatiles inside of the icy satellites and the KBO objects is based (i) on the calculation of the impact-originated increase of temperature of the volatile-bearing grains of accreting material, and (ii) on the thermodynamic properties of the volatiles under consideration. It is found that the highly volatile materials are accumulated in the deep interiors and the less volatile are expected to survive everywhere, including the outer layers. The particular calculations are performed for Saturnian and Uranian icy satellites (from Epimetheus to Titania, with radii $65-780 \mathrm{~km}$ ) as well as for some of $\mathrm{KBO}$ objects. Considered volatiles are $\mathrm{H}_{2} \mathrm{O}, \mathrm{CO}_{2}, \mathrm{CO}, \mathrm{NH}_{3}, \mathrm{CH}_{4}, \mathrm{~N}_{2}$, and $\mathrm{C}_{2} \mathrm{H}_{6}$.
\end{abstract}

\section{Minor Body Occultations: Statistics and Survey Requirements Asantha Cooray (California Institute of Technology, CA, USA)}

\footnotetext{
Abstract. The occultation of background stellar sources by foreground minor bodies, mainly Kuiper Belt Objects (KBOs), can be used to survey physical properties of the foreground population. We will discuss statistics related to $\mathrm{KBO}$ occultations and discuss what constraints can be placed on the KBO population. While the optical depth for occultation is relatively high for KBOs, lowering the number of stars one should monitor, the event duration is at the
} 
subsecond level and puts strong constraints on the instrumentation side. We discuss requirements for a reasonable ground-based survey and suggest that an array of telescopes, spread over few kilometers with imaging and timing capabilities at the level of 100 millisecond or less down to a magnitude limit of 14 in the $\mathrm{V}$-band, may be used to probe the KBO population below a kilometer in size. With an array, one also probes the projected shapes of KBOs and the presence of binaries. We also discuss relative merits of a space-based experiment. If fainter stars are monitored, the diffraction fringes present in certain light curves can be used to extract physical information related to the foreground occulting body.

\title{
Existence of the Lunar Core
}

\section{Alexander Gusev \& Natasha Petrova (Kazan State University, Russia)}

\begin{abstract}
The question of lunar core existence is still an open question. If core existence will be verified by future direct measurements, such a core would give constraints to the model of origin of the Moon. A short review of results concerning facts of core existence is given in the report. The most advances in this study were obtained as results of the investigation of Lunar gravity and physical libration. The accumulated data about the lunar gravity are now very wealthy, but there is still a great deal to supplement the data about gravity and topography picture on the far-side. Though there are many inferential evidence of presence at the Moon of a core, nevertheless the direct experiments on detection of the core and determination of its characteristics are very important. The large prospects in the decision of this problem are opened by the RISE and ILOM projects of SELENE I, II mission. Obtained data will allow to improve the physical libration theory of the Moon and together with theoretical and observational libration data they will provide a further study of the lunar interior and, as a consequence, of its origin and evolution.
\end{abstract}

\section{Centrifugal Librations due to Lunar Core-Mantle Couplings Eric Bois (Observatoire de Bordeaux L3AB, UMR CNRS/INSU 5804, France)}

\begin{abstract}
We present a study of the dynamical behavior of a molten core inside the Moon related to the mantle by inertial coupling. In order to integrate the lunar core-mantle interaction in a realistic model of the Moon's rotation, we have used our SONYR (acronym of Spin-Orbit N-bodY Relativistic) model of the solar System including the Moon's spin-orbit motion. This model was previously built in accordance with the requirements of the Lunar Laser Ranging observational accuracy. We have extended this model to the spin-orbit couplings of the terrestrial planets in order to compare different dynamical behaviors of core-mantle interactions in these planets (Mercury, Venus, Earth and Mars).

Our core-mantle mechanism prove to be adequate to excite the two resonant frequencies of the lunar physical librations, namely 2.9 and 80.1 years. ignature of such a core with for instance a $1 / 10$ homothetic ratio appears clearly on the proper rotation angle; the amplitude is then around 12 milli-arcseconds and its period 2.9 years.
\end{abstract}


Besides, we present the results obtained for various lunar nucleus radii and various initial nutations of the core relatively to the mantle. Other computations and comparisons are in progress involving Mercury, the Earth and Mars.

\title{
Mid-Infrared Spectrophotometry of Saturn's Ring System
}

Padma A. Yanamandra-Fisher, Glenn S. Orton \& Brendan M. Fisher (Jet Propulsion Laboratory, Pasadena, CA, USA)

\begin{abstract}
We observed Saturn's main rings at the NASA/InfraRed Telescope Facility (IRTF) from 1995 to 2003 with MIRLIN, a 10-micron camera at several diagnostic wavelengths in the 8-to 24 -micron spectral window, covering one fourth of a Saturnian year, and followed the progressive march of the rings from their edge-on presentation at the equator in 1995 to their maximum opening, obscuring the northern pole of Saturn. Our preliminary results indicate that the brightness temperature of the rings peaks near 18 microns. There also exists an asymmetry between the East and West ansae of few degrees. This is similar to the near-infrared albedo asymmetry between the ansae and reflectivities at visible wavelengths. Our current efforts are aimed at modelling the ring opacities in the mid-infrared as function of changes in solar elevation angle, inclination and phase angles of the rings. In particular, the A ring had changed from being edge on in 1994 to completely unshadowed in 2003. These observations will provide constraints on thermal asymmetries and opacity of the ring. Current thermal models will be validated against these observations and previous data sets acquired by Voyager and ISO and data to be acquired by Cassini and possibly SIRTF and SOFIA.
\end{abstract}

